\title{
Optimization of capsid-incorporated antigens for a novel adenovirus vaccine approach
} \author{
Qiana L Matthews ${ }^{1}$, PingAr Yang', Qi Wu2 ${ }^{2}$, Natalya Belousova ${ }^{3}$,
} Angel A Rivera ${ }^{1}$, Mariam A Stoff-Khalilii ${ }^{4}$, Reinhard Waehler ${ }^{1}$, HuiChen $\mathrm{Hsu}^{2}$, Zan $\mathrm{Li}^{5}$, Jing $\mathrm{Li}^{1}$, John D Mountz ${ }^{2,6}$, Hongju $\mathrm{Wu}^{1}$ and David T Curiel ${ }^{* 1}$

\begin{abstract}
Address: ${ }^{1}$ Division of Human Gene Therapy, Departments of Medicine, Pathology, Surgery, Obstetrics and Gynecology, and the Gene Therapy Center, University of Alabama at Birmingham, Birmingham, USA, ${ }^{2}$ Division of Clinical Immunology and Rheumatology, Department of Medicine, University of Alabama at Birmingham, Birmingham, USA, ${ }^{3}$ Department of Experimental Diagnostic Imaging, MD Anderson Cancer Center, University of Texas, Houston, USA, ${ }^{4}$ Department of Obstetrics and Gynecology, University of Duesseldorf, Medical Center, Duesseldorf, Germany, ${ }^{5}$ Alabama School of Fine Arts, Birmingham, USA and ${ }^{6}$ Birmingham VA Medical Center, Birmingham, USA

Email: Qiana L Matthews - qlm@uab.edu; PingAr Yang - shuang@uab.edu; Qi Wu - qiwu@uab.edu; Natalya Belousova - Natalya.Belousova@di.mdacc.tmc.edu; Angel A Rivera - aarivera@uab.edu; Mariam A Stoff-Khalili - mariam.stoffkhalili@uk-koeln.de; Reinhard Waehler -waehler@uab.edu; Hui-Chen Hsu - HuiChen.Hsu@ccc.uab.edu; Zan Li - parisintherain@gmail.com; Jing Li - jili@uab.edu; John D Mountz - John.Mountz@ccc.uab.edu; Hongju Wu - hongjuwu@uab.edu; David T Curiel* - curiel@uab.edu

* Corresponding author
\end{abstract}

Published: 2I August 2008

Virology Journal 2008, 5:98 doi:10.1186/1743-422X-5-98

This article is available from: http://www.virologyj.com/content/5/I/98

(c) 2008 Matthews et al; licensee BioMed Central Ltd.

This is an Open Access article distributed under the terms of the Creative Commons Attribution License (http://creativecommons.org/licenses/by/2.0), which permits unrestricted use, distribution, and reproduction in any medium, provided the original work is properly cited.
Received: 19 June 2008

Accepted: 2I August 2008

\begin{abstract}
Despite the many potential advantages of Ad vectors for vaccine application, the full utility of current $A d$ vaccines may be limited by the host anti-vector immune response. Direct incorporation of antigens into the adenovirus capsid offers a new and exciting approach for vaccination strategies; this strategy exploits the inherent antigenicity of the Ad vector. Critical to exploiting Ad in this new context is the placement of antigenic epitopes within the major Ad capsid protein, hexon. In our current study we illustrate that we have the capability to place a range of antigenic epitopes within Ad5 capsid protein hexon hypervariable regions (HVRs) 2 or 5 , thus producing viable Ad virions. Our data define the maximal incorporation size at HVR2 or HVR5 as it relates to identical antigenic epitopes. In addition, this data suggests that Ad5 HVR5 is more permissive to a range of insertions. Most importantly, repeated administration of our hexon-modified viruses resulted in a secondary anti-antigen response, whereas minimal secondary effect was present after administration of Ad5 control. Our study describes antigen placement and optimization within the context of the capsid incorporation approach of Ad vaccine employment, thereby broadening this new methodology.
\end{abstract}

\section{Introduction}

Adenoviruses (Ad) have recently been employed for a wide range of vaccination strategies [1]. In this regard, a number of practical advantages are recognized in using Ad-based vectors for antigen gene delivery. These advan- tages include the ease of manipulation of the viral genome, the ability to prepare high titer stocks of recombinant virions, and the ability of the vector to infect a wide array of target cells [2-4] relevant to the achievement of a useful vaccine effect. These considerations highlight the 
emerging recognition that Ad vectors embody enormous promise for the realization of diverse vaccine interventions. Of note, Ad-based vaccinations have been practically translated for human applications and have progressed in a variety of immunization contexts such as cancer and infectious diseases [5-12].

Currently, new methods to exploit Ad for vaccine purposes have been developed. These recent approaches have utilized the natural mechanisms of Ad virion immunogenicity whereby antigen epitopes can be directly incorporated into the viral capsid as the basis by which immune presentation of the epitope is achieved [10,13-16]. Strategies advancing this "capsid incorporation" paradigm have evaluated a range of virion capsid proteins as well as a variety of antigens, model and pathogenic [10,14-17].

The major capsid protein hexon has been the focus of the majority of these capsid incorporation strategies owing to its natural role in the generation of anti-Ad immune response and its numerical representation vis a via the virion's structural organization $[14,18]$. Using this strategy, we have developed the means to incorporate heterologous peptide epitopes specifically within the major surface-exposed domains of the Ad capsid protein hexon [18]. Of note, our previous studies have show that we can incorporate small heterologous peptides into Ad hexon hypervariable regions (HVRs) without perturbing viral viability and major biological characteristics such as replication, thermostability, or native infectivity [18]. Other published studies have focused on incorporations at HVR5 or single site incorporations [17]. However, it has been recognized that the ability to place antigen within multiple sites of the hexon capsid protein holds important potential for presenting multiple epitopes/antigens or several copies of the same epitope within a single Ad vector-based vaccine.

In this regard, capsid surface localization of HVR sites derived from X-ray crystallography suggests that both HVR2 and HVR5 loci are potentially useful for capsid incorporation of antigens for vaccination. As noted, there have been recent reports in which HVR5 has been exploited with respect to epitope insertion $[10,14,15,18$ 21]. Based on our abilities to manipulate both HVR2 and HVR5 sites, we sought to explore the relative merits of these two hexon locales. To compare the flexibility and capacities of HVR2 and HVR5, respectively we genetically incorporated identical epitopes of incrementally increasing size within HVR2 or HVR5 of Ad5 hexon. Our study illustrates that hexon incorporated model antigens elicit a varied immune response in the context of antigen placement or antigen size at both the HVR2 or HVR5 locales.

\section{Materials and methods Antibodies}

Mouse anti-penta-His ${ }_{6}$ tag monoclonal antibody (34660) was purchased from Qiagen (Valencia, CA). Horse radish peroxidase (HRP)-conjugated goat anti-mouse secondary antibodies were purchased from DakoCytomation (Denmark).

\section{Cell culture}

Human embryonic kidney cells (293) were obtained from and cultured in the medium recommended by the American Type Culture Collection (Manassas, VA). All cell lines were incubated at $37^{\circ} \mathrm{C}$ and $5 \% \mathrm{CO}_{2}$ under humidified conditions.

\section{Recombinant adenovirus construction}

In order to generate recombinant adenoviruses with hexon insertions of arginine-glycine-aspartic acid (RGD)containing sequences, fragments of the Ad5 penton base gene corresponding to the RGD motifs were derived by PCR and cloned into the BamHI site in the previously described HVR2-His $/$ /pH5S or HVR5-His 6 /pH5S plasmids [18]. The sequences corresponding to penton basederived peptides, 33RGD, 43RGD, 53RGD, 63RGD, 73RGD, and 83RGD, were PCR amplified from Ad5 genomic DNA with the following pairs of primers: 33RGD sense (s) and 33RGD anti-sense (as), 43RGD sense (s) and 43RGD anti-sense (as), 53RGD sense (s) and 53RGD anti-sense (as), 63RGD sense (s) and 63RGD anti-sense (as), 73RGD sense (s) and 73RGD anti-sense (as), and 83RGD sense (s) and 83RGD anti-sense (as) (Table 1). For additional details, see reference [22]. To create Ad5

Table I: Primers used in this study.

\begin{tabular}{ll}
\hline 33RGD -(as) & CGGGATCCTGCTTCGGCCTCAGCGCGC \\
33RGD -(s) & CGGGATCCGCCGCGGCAATGCAGCC \\
43RGD -(as) & CGGGATCCGGCAGCTTCGGCCGCTG \\
43RGD -(a) & CGGGATCCAACTCCAACGCGGCAGCC \\
& \\
53RGD -(as) & CGGGATCCTTGCGCAGCGGGGGC \\
53RGD -(a) & CGGGATCCAGCGGCGCGGAAGAGAACTC \\
63RGD -(as) & CGGGATCCCTTCTCGACCTCGGGTTGCG \\
63RGD -(a) & CGGGATCCAGCAACAGCAGTGGCAGCG \\
73RGD -(as) & CGGGATCCCGGTTTCTTCTGAGGCTTCTCG \\
73RGD -(a) & CGGGATCCGGTGGCGCAGGCGG \\
& \\
83RGD -(as) & CGGGATCCCAGGGGTTTGATCACCGGTTT \\
83RGD -(a) & CGGGATCCACCGAACAGGGCGGGG \\
3'HVR5-(as) & GGCATGTAAGAAATATGAGTGTCTGGG \\
5'HVR2-(s) & CTCACGTATTTGGGCAGGCGCC
\end{tabular}

\footnotetext{
a antisense, as; sense, $s$.

b Underlined letters represent the sequences

encoding the RGD motifs.
} 
vectors containing RGD epitopes in the HVRs of hexon, these resulting plasmids were digested with EcoRI and PmeI. These resulting fragments containing the homologous recombination regions and the hexon genes were purified, then recombined with a SwaI-digested Ad5 backbone vector that lacks the hexon gene, pAd5/ $\Delta \mathrm{H} 5$ [23]. These recombination reactions were performed in Escherichia coli BJ5183 (Strategene, La Jolla, CA). The resultant clones were designated Ad5/HVR2-33RGD-His ${ }_{6}$, Ad5/HVR5-33RGD-His ${ }_{6}$, Ad5/HVR5-43RGD-His ${ }_{6}$, and Ad5/HVR5-53RGD-His ${ }_{6}$, all of which contain the green fluorescence protein gene and firefly luciferase gene in the E1 region [18]. The constructs were confirmed by restriction enzyme digestions and sequence analysis. Ad5, Ad5/ HVR2-His $_{6}$, and Ad5/HVR5-His 6 were previously constructed as described [18].

\section{Virus rescue and preparation}

To rescue viruses, the constructed plasmids were digested with PacI, and $2 \mu \mathrm{g}$ DNA were transfected (Lipofectamine 2000 Reagent, Invitrogen, Carlsbad, CA) into the Ad-E1expressing 293 cells. After plaques formed, they were processed for large-scale proliferation in 293 cells. Viruses were purified by double cesium chloride ultracentrifugation and dialyzed against phosphate-buffered saline containing $10 \%$ glycerol. Viruses were stored at $-80^{\circ} \mathrm{C}$ until use. Final aliquots of virus were analyzed for physical titer using absorbance at $260 \mathrm{~nm}$. The infectious viral titer (infectious particles per $\mathrm{ml}$ ) was determined by tissue culture infectious dose $\left(\mathrm{TCID}_{50}\right.$ ) assay. Modifications of the hexon gene was confirmed by PCR analysis with the primers 5'HVR2 (s), CTCACGTATTTGGGCAGGCGCC and 3'HVR5(as), GGCATGTAAGAAATATGAGTGTCTGGG, which anneal up and downstream of the site of the insertion within the hexon open reading frame (Table 1).

\section{Whole virus ELISA and sera ELISA}

The enzyme-linked immunosorbent assay (ELISA) assay was performed essentially as described previously [24]. In brief, different amounts of viruses ranging from $4 \times 10^{6}$ to $9 \times 10^{9}$ VPs were immobilized in wells of a 96-well plate (Nunc Maxisorp, Rochester, NY) by overnight incubation in (per well) $100 \mu \mathrm{l}$ of $100 \mathrm{mM}$ carbonate buffer ( $\mathrm{pH}$ 9.5) at $4{ }^{\circ} \mathrm{C}$. After washing with $0.05 \%$ Tween 20 in Tris-buffered saline and blocking with blocking solution (2\% bovine serum albumin and $0.05 \%$ Tween 20 in TBS), the immobilized viruses were incubated with anti-penta-His ${ }_{6}$ tag monoclonal antibody (Qiagen, Valencia, CA) for $2 \mathrm{~h}$ at room temperature, followed by AP-conjugated goat anti-mouse antibody incubation. Colormetric reaction was performed with p-nitrophenyl phosphate (SigmaAldrich, St. Louis, $\mathrm{MO}$ ) as recommended by the manufacturer, and optical density at 450-650 nm ( $\left.\mathrm{OD}_{450-650}\right)$ was obtained with a microplate reader (Molecular Devices).
For the anti-RGD33-His ${ }_{6}$ and anti-His ${ }_{6}$ response, ELISA plates (Nunc Maxisorp, Rochester, NY) were coated with $20 \mu \mathrm{M}$ of the RGD33-His ${ }_{6}$ peptide or the $\mathrm{His}_{6}$ peptide in $100 \mu \mathrm{l}$ of $50 \mathrm{mM}$ carbonate ( $\mathrm{pH}$ 9.6) per well according to the method we described previously [25]. Plates were washed and then blocked with 3\% BSA/PBS. After washing, $60 \mu \mathrm{l}$ of 1:50 diluted sera was added. After incubation for at least $2 \mathrm{hr}$ at RT, the plates were extensively washed, and the isotype-specific HRP-conjugated anti-mouse antibody (Southern Biotech., Birmingham, AL) was added. ELISAs were developed with TMB substrate (SigmaAldrich, St. Louis, MO). OD ${ }_{450-650}$ was measured on an Emax microplate reader.

\section{Mouse immunization}

Female $\mathrm{C} 57 \mathrm{BL} / 6 \mathrm{~J}\left(\mathrm{H}-2^{\mathrm{b}}\right)$ mice at 6-8 weeks of age were obtained from the Jackson Laboratory (Bar Harbor, ME). Groups of at least three to five mice were analyzed in each experiment or at each time point. For antibody response analysis, the following adenoviral vectors were injected into each group of mice: Ad5, Ad5/HVR2-His ${ }_{6}$ Ad5/ HVR5-His $_{6}$, Ad5/HVR2-33RGD-His ${ }_{6}$, Ad5/HVR5-33RGDHis $_{6}{ }$ Ad5/HVR5-43RGD-His ${ }_{6}$, and Ad5/HVR5-53RGDHis $_{6}$ at $1 \times 10^{10}$ viral particles (VPs) per mouse using tail intravenous injection. For CD4 ${ }^{+} \mathrm{T}$ cell response analysis, the following adenoviral vectors were injected to each group of mice: Ad5, Ad5/HVR2-33RGD-His ${ }_{6}$, or Ad5/ HVR5-33RGD-His $_{6}$ at $1 \times 10^{10} \mathrm{VP}$ per mouse using tail intravenous injection. On day 40 , these mice were intravenously boosted with the same dose of the same vectors or peptide. These mice were then sacrificed 9 days later. All animal protocols were approved by the Institutional Animal Care and Use Committee at the University of Alabama at Birmingham.

\section{Peptide prediction and synthesis}

The antigenic epitope of $\mathrm{His}_{6}$ and RGD33-His ${ }_{6}$ were predicted using the Emboss program http://emboss.source forge.net/apps/antigenic.html and by the Kyle-Doolittle hydropathic plot from the FIMM database of functional molecular immunology http://sdmc.lit.org.sg:8080/ fimm/. Peptide sequences that were given high binding scores in both prediction programs were chosen for ELISA analysis. Peptides were synthesized by GenScript Co (Piscataway, NJ) and were $>98 \%$ pure as indicated by analytical high-performance liquid chromatography. Peptides were dissolved in $100 \%$ DMSO at a concentration of 10 $\mathrm{mM}$ and stored at $-20^{\circ} \mathrm{C}$ until use.

\section{Intracellular flow cytometry staining}

Intracellular analysis of cytokines produced by CD4+ T cells was carried out using FACS analysis according to the protocol of Harrington, et al. and Mangan, et al.[26,27]. Briefly, prior to carrying out intracellular cytokine staining, polarized whole spleen cells or CD4+ T cells were 
stimulated for $5 \mathrm{~h}$ with phorbylmyristyl acetate $(50 \mathrm{ng} /$ $\mathrm{ml}$; Sigma-Aldrich, St. Louis, MO) and ionomycin (750 $\mathrm{ng} / \mathrm{ml}$; Sigma-Aldrich) in the presence of either GolgiStop at the recommended concentrations (BD Pharmingen, San Diego, CA). Cells were first stained extracellularly with fluorescein isothiocyanate-conjugated anti-CD4+ (RM4-5), fixed and permeabilized with Cytofix/Cytoperm solution (BD Pharmingen), and then stained intracellularly with allophycocyanin-conjugated anti-IFN- $\gamma$ (XMG1.2) and anti-IL-4 (11B11). Samples were acquired on a FACSCalibur (Becton Dickinson, Franklin Lakes, NJ) and data were analyzed with FlowJo (Ashland, OR) software.

\section{Statistical evaluation}

The data are presented as the mean \pm the standard error. Statistical analyses were performed with the nonpaired two-tailed Student $t$ test, assuming equal variance. Statistical significance was defined as $P<0.05$.

\section{Results \\ Incorporation of antigenic epitopes within Ad5 hexon HVR2 or HVR5}

In order to assess the capacity of the Ad5 hexon hypervariable regions to accommodate heterologous polypeptides, we genetically incorporated incrementally increasing fragments of the Arg-Gly-Asp (RGD)-containing loop of the Ad5 penton base. Fragments were engineered to contain the RGD motif in the middle, flanked by penton basederived sequences of equal lengths on both sides. The length of each flanking sequence in the shortest construct was 15 amino acid (aa) residues; this was increased by 10aa increments in succeeding constructs [22]. DNA sequences corresponding to the fragments of the penton base protein were assembled by PCR (Figure 1A. and Table 1). These PCR products were cloned between codons for Ser192 and His193 (Fig. 1B-1) of the previously modified Ad5/HVR2-His ${ }_{6}$ genome [18] or between codons for Ser273 and His274 of the previously modified Ad5/HVR5-His ${ }_{6}$ genome (Fig. 1B-2) [18]. A total of six fragments encoding the penton base protein ranging in size from 33, 43, 53, 63, 73, and 83 aa were amplified and incorporated into the Ad5 hexon HVR2 or HVR5 region.

\section{Ad5 hexon HVR2 or HVR5 can accommodate large heterologous polypeptides}

The newly designed hexon genes were transferred into the E1-deleted Ad5 genome lacking the hexon gene. Subsequent transfection of 293 cells with the resultant recombinant genomes led to the rescue of 4 of the 12 vectors. Viable viruses were produced with incorporation of 33 aa plus a 12 aa linker at HVR2 or HVR5 (Table 2A). In addition, viable viruses were rescued with incorporations of 43 and 53 aa plus linkers at HVR5 (Table 2A). The recombinant hexon viruses rescued contained the aforemen-
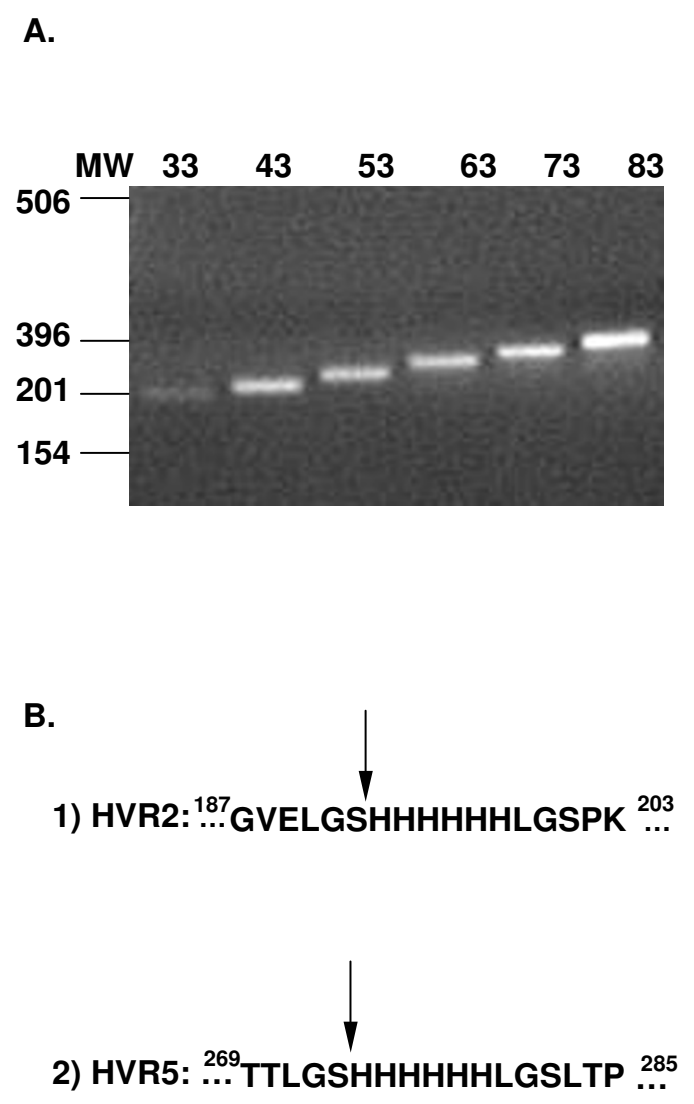

Figure I

Construction of hexon modified genomes. (A) Agarose gel electrophoresis of PCR products were obtained through PCR of genomic DNA of Ad5 using epitope specific primers (Table I). (B) HVR2 or HVR5 shuttle vector sites which were modified with a RGD motif. The DNA encoding for the respective RGD motifs were cloned into the HVRs of our previously modified shuttle vectors within the LGSHHHHHLGS linker, as indicated by the arrows.

tioned penton base-RGD composition (Table 2B). Rescued viruses were further amplified and their identities were confirmed by PCR, non-defective revertant viruses were not detected (data not shown). These data were further confirmed by partial sequencing of the hexon genes contained in the Ad5/HVR2 or Ad5/HVR5 genomes. Having established the identities of the newly rescued Ad viruses, we next tested whether the large incorporations in hexon had any effect on virus stability and/or infectious properties. Physical titer, as well as infectious titer was determined for each virus. The viral particle/infectious particle (VP/IP) ratio was calculated for the control viruses, (Ad5, Ad/HVR2-His ${ }_{6}$, and Ad/HVR5-His ${ }_{6}$ ) as well as all of the hexon-modified viruses. We observed that, as the incorporation size at hexon increased the VP/IP ratio 
Table 2: Viable viruses.

\begin{tabular}{|c|c|c|}
\hline \multicolumn{3}{|l|}{ A. } \\
\hline 33RGD Motif + 12 aa Linker & + & + \\
\hline 43RGD Motif + 12 aa Linker & - & + \\
\hline 53RGD Motif + 12 aa Linker & - & + \\
\hline 63RGD Motif + 12 aa Linker & - & - \\
\hline 73RGD Motif + 12 aa Linker & - & - \\
\hline 83RGD Motif + 12 aa Linker & - & - \\
\hline \multicolumn{3}{|c|}{$(+)=$ viable viruses $(-)=$ not viable viruses. } \\
\hline \multicolumn{3}{|l|}{ B. } \\
\hline 33RGD motif- & \multicolumn{2}{|c|}{ AAAMQPVEDMNDHAIRGDTFATRAEEKRAEAEA } \\
\hline 43RGD motif- & \multicolumn{2}{|c|}{ NSNAAAAAMQPVEDMNDHAIRGDTFATRAEEKRAEAEAAAEAA } \\
\hline 53RGD motif- & \multicolumn{2}{|c|}{ SGAEENSNAAAAAMQPVEDMNDHAIRGDTFATRAEEKRAEAEAAAEAAAPAAQ } \\
\hline 63RGD motif- & \multicolumn{2}{|c|}{ SNSSGSGAEENSNAAAAAMQPVEDMNDHAIRGDTFATRAEEKRAEAEAAAEAAAPAAQPEVEK } \\
\hline 73RGD motif- & \multicolumn{2}{|c|}{$\begin{array}{l}\text { GGAGGSNSSGSGAEENSNAAAAAMQPVEDMNDHAIRGDTFATRAEEKRAEAEAAAEAAAPAAQ } \\
\text { PEVEKPQKKP }\end{array}$} \\
\hline 83RGD motif- & \multicolumn{2}{|c|}{$\begin{array}{l}\text { TEQGGGGAGGSNSSGSGAEENSNAAAAAMQPVEDMNDHAIRGDTFATRAEEKRAEAEAAAEAA } \\
\text { APAAQPEVEKPQKKPVIKPL }\end{array}$} \\
\hline
\end{tabular}

Core Arg-Gly-Asp (RGD) motif italicized and underlined

also increased compared to the $\mathrm{His}_{6}$ vectors or unmodified Ad5 (Table 3). A normal VP/IP ratio of unmodified Ad ranges from $~ 10-30$.

\section{Large epitope incorporations are accessible within Ad5 hexon HVR2 or HVR5}

Our previous studies determined that $\mathrm{His}_{6}$ epitopes incorporated in HVR2 or HVR5 could bind to anti-His ${ }_{6}$ tag antibody via an ELISA assay, therefore surface exposed [18]. After establishing the ability to place large epitopes into HVR2 or HVR5, we next sought to explore whether the larger epitope incorporations were also surface exposed. Only surface expressed motifs should be accessible to antibody binding, thus, we verified that the RGD-His 6 motif in HVR2 or HVR5 were accessible on the virion surface by ELISA with an anti-His ${ }_{6}$ antibody (Fig. 2). In the assay, varying amounts of purified viruses were immobilized in the wells of ELISA plates and incubated with antiHis $_{6}$ antibody and appropriate secondary antibody. The results demonstrated that Ad5/HVR2-33RGD-His ${ }_{6}$, Ad5/
HVR5-33RGD-His $_{6}$, Ad5/HVR5-43RGD-His ${ }_{6}$, Ad5/HVR553RGD-His ${ }_{6}$, and positive controls (Ad5/HVR2-His ${ }_{6}$ and Ad5/HVR5-His ${ }_{6}$ ) [18] have significant levels of binding by anti-His ${ }_{6}$ antibody, while negative control Ad5 showed essentially no binding. These results indicate that the RGD-His $_{6}$ epitopes incorporated in HVR2 or HVR5 are exposed on the virion surface.

\section{Incorporation of epitopes within Ad5 hexon HVR2 or HVR5 elicits an IgG immune response}

We next sought to establish that these modified Ad vectors could elicit an immune response in mice. In this regard, a high IgG response is in part indicative of protection for the host organism. Equal amounts of viral particles were used to immunize C57BL/6J mice. The sera from these mice were collected at multiple time points up to 70 days post-injection for analysis with ELISA binding assays (Fig. 3 ). For these assays synthesized His ${ }_{6}$ peptides (His6/ linker) (LGSHHHHHHLGS) were first bound to the ELISA plate, the plates were then incubated with immu-

Table 3: We observed that, as the incorporation size at hexon increased the VP/IP ratio also increased compared to the His6 vectors or unmodified Ad5 .

\begin{tabular}{llll}
\hline Modified Viruses & Viral Particle (VP) & Infectious Particles (IP) & VP/IP \\
\hline Ad5 & $4.58 \times 10^{12} \mathrm{vp} / \mathrm{ml}$ & $3 \times 10^{11} \mathrm{PFU} / \mathrm{ml}$ & 15.26 \\
Ad/HVR2-His & $5 \times 10^{12} \mathrm{vp} / \mathrm{ml}$ & $3 \times 10^{11} \mathrm{PFU} / \mathrm{ml}$ & 14.7 \\
Ad/HVR5-His & $5 \times 10^{12} \mathrm{vp} / \mathrm{ml}$ & $4 \times 10^{11} \mathrm{PFU} / \mathrm{ml}$ & 14.25 \\
Ad/HVR2-33RGD-His & $2.7 \times 10^{11} \mathrm{vp} / \mathrm{ml}$ & $1.58 \times 10^{9} \mathrm{PFU} / \mathrm{ml}$ & 236 \\
Ad/HVR5-33RGD-His & $1.85 \times 10^{12} \mathrm{vp} / \mathrm{ml}$ & $3.98 \times 10^{8} \mathrm{PFU} / \mathrm{ml}$ & 1,170 \\
Ad/HVR5-43RGD-His & $2.35 \times 10_{6}^{12} \mathrm{vp} / \mathrm{ml}$ & $1.25 \times 10^{9} \mathrm{PFU} / \mathrm{ml}$ & 5,940 \\
Ad/HVR5-53RGD-His & $1.01 \times 10^{12} \mathrm{vp} / \mathrm{ml}$ & 808
\end{tabular}




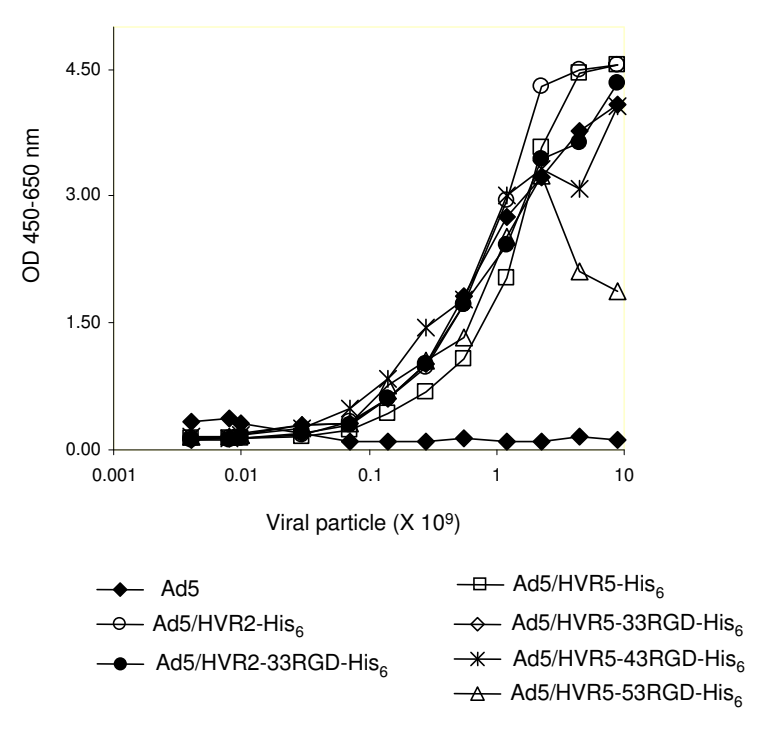

Figure 2

Model epitopes incorporated in HVRs are accessible in the context of an intact virion. In the assay, varying amounts of purified viruses were immobilized in the wells of ELISA plates and incubated with anti-His 6 tag antibody. The binding was detected with an AP-conjugated secondary antibody. These results suggested that the model antigens (tagged with $\mathrm{His}_{6}$ epitopes) and the $\mathrm{His}_{6}$ epitopes (controls) incorporated into HVR2 or HVR5 were accessible to anti$\mathrm{His}_{6}$ tag antibody at the virion level, indicating that the epitopes were exposed on the virion surface. All of the Ad vectors except Ad5 present $\mathrm{His}_{6}$ or RGD-His 6 within the hexon. The $\mathrm{His}_{6}$ antigenic peptide is presented by $\mathrm{Ad} 5 /$ HVR5-His 6 and Ad5/HVR5-His 6 .

nized mice sera. The binding of mouse anti-His ${ }_{6}$ IgG to the synthesized peptides was detected with a HRP-conjugated secondary antibody (Fig. 3A-B). The data illustrate no binding of the $\mathrm{His}_{6}$ peptide with serum from the mice immunized with the negative control Ad5 or the uninfected mice. This is in contrast to substantial binding seen with mice immunized with Ad5/HVR5-43RGD-His ${ }_{6}$ Ad5/HVR5-53RGD-His ${ }_{6}$, or positive controls [18], while Ad5/HVR2-33RGD-His ${ }_{6}$ and Ad5/HVR5-33RGD-His showed weaker binding. Of note, all of these vectors contain $\mathrm{His}_{6}$ epitopes in the modified HVR regions. The data demonstrated that immunization with Ads containing capsid-incorporated antigen elicits an anti-His ${ }_{6}$ IgG response in mice which shows a substantial peak at 30 days (Fig. 3A) when observed over a time course of 70 days (Fig. 3B); furthermore, the magnitude of the immune response was a function of antigen locale and flanking sequences (Fig. 3A-B).
Similarly, significant results were seen in response to the synthesized 33 RGD-His ${ }_{6}$ antigenic peptide (which contains a core RGD residue flanked by $\mathrm{His}_{6} /$ linker). These results were observed at time points ranging from 1-70 days (Fig. 4A). Since the probe contained both the 33RGD and $\mathrm{His}_{6}$ epitopes binding was expected for all of the sera samples except sera from Ad5 immunized mice.

\section{Incorporation of epitopes within Ad5 hexon HVR2 or HVR5 elicits a variable humoral immune response}

We next performed experiments to determine the quantitative aspects of the isotype-specific humoral responses that were generated in response to our vectors. For IgG1 isotype antibodies, the highest levels of anti-33RGD-His IgG1 were seen on day 7 after immunization with Ad5/ HVR5-33RGD-His $_{6}$, Ad5/HVR5-43RGD-His ${ }_{6}$, and Ad5/ HVR5-53RGD-His $_{6}$ virions. These results confirm that the HVR5 loop provides the most immunogenic environment for production of anti-33RGD-His ${ }_{6}$ IgG1 isotype antibodies. Further supporting this, the IgG1 antibody response to the $33 \mathrm{RGD}-\mathrm{His}_{6}$ in the HVR2 loop was markedly lower when directly compared to the 33RGD-His ${ }_{6}$ in the HVR5 loop (Fig. 4B). The IgG2b (Fig. 4C) and IgG2c (Fig. 4D) isotype specific antibody response to RGD33-His ${ }_{6}$ epitope followed the same pattern as IgG1, except that peak values did not occur until day 12 after immunization, and antibody levels were sustained at high levels out to day 50 . These results indicate that RGD-His ${ }_{6}$ epitopes in the HVR5 loop are more immunogenic and invoke higher sera levels of total anti-33RGD-His ${ }_{6}$ IgG antibodies than RGD-His ${ }_{6}$ epitopes in the HVR2 loop.

\section{Incorporation of epitopes within Ad5 hexon HVR2 or HVR5 elicits a varied $T$ cell and secondary response}

Increased antibody titers of the IgG class require help from either Th1 CD4+ $\mathrm{T}$ cells that produce IFN- $\gamma$ or Th2 $\mathrm{CD}^{+}{ }^{+} \mathrm{T}$ cells that produce IL-4 [28]. Th1 is generally associated with isotype class switching to $\operatorname{IgG} 2 \mathrm{a}\left(\mathrm{in} \mathrm{IgH}^{\mathrm{d}}\right.$ strain of mice) or IgG2c (in IgH $^{b}$ stain), whereas Th2 help is associated with class switching to IgG1 or IgG2b in mice [29]. To determine if there is an increase in Th1 or Th2 response to the $33 \mathrm{RGD}-\mathrm{His}_{6}$ peptide after boost of the Ad5/HVR2-33RGD-His ${ }_{6}$ or Ad5/HVR5-33RGD-His ${ }_{6}$ vector, a single-cell suspension of spleen cells was prepared on day 9 after secondary virus infection. Cells were stained with a fluorescent labeled anti-CD4 antibody and then permeabilized in intracellular stain with fluorescent conjugated antibodies against IL- 4 or IFN- $\gamma$. CD4 ${ }^{+} \mathrm{T}$ cells from mice immunized with Ad5/HVR5-33RGD-His ${ }_{6}$ produced a significant increase in IFN- $\gamma$ expressing cells and a lesser increase in CD4+ $\mathrm{T}$ cells that express IL-4. In C57BL/6J mice immunized with Ad5/HVR2-33RGD-His 6 or Ad5, there were very low numbers of IFN $-\gamma^{+} \mathrm{CD} 4{ }^{+} \mathrm{T}$ cells (Fig. $5 \mathrm{~A}-\mathrm{B})$. CD4 ${ }^{+}$cells expressing IL-4 was equally increased in mice immunized with Ad5/HVR2-33RGD $\mathrm{His}_{6}$ or with 
A.

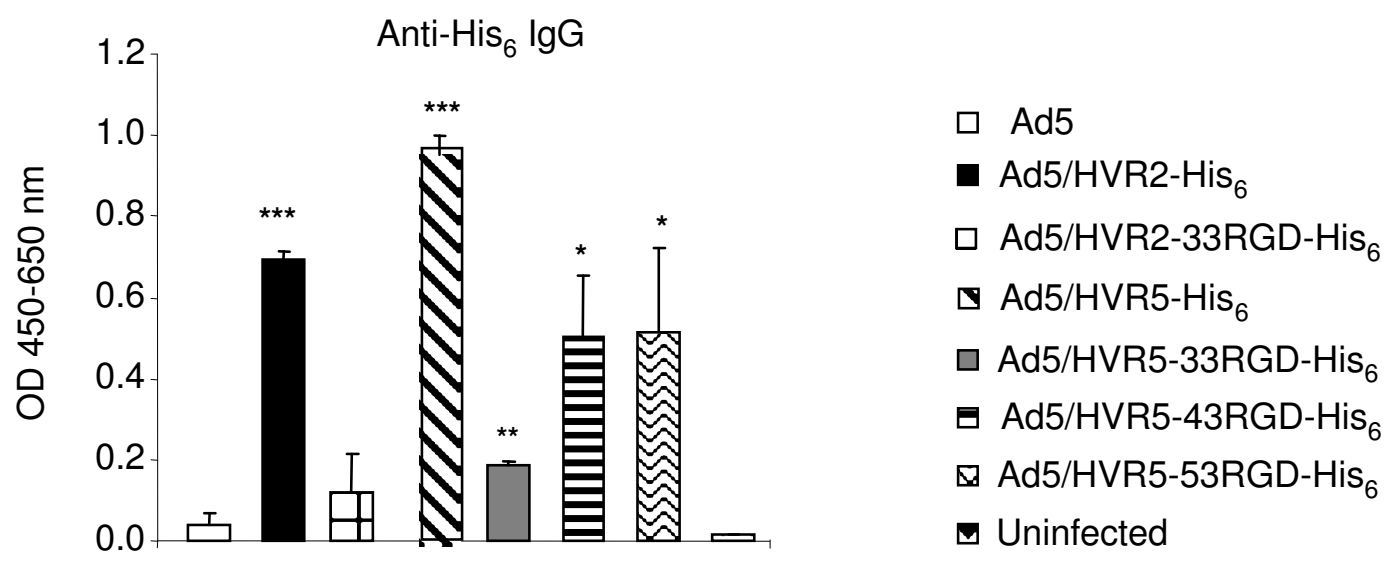

Day 30 post-injection

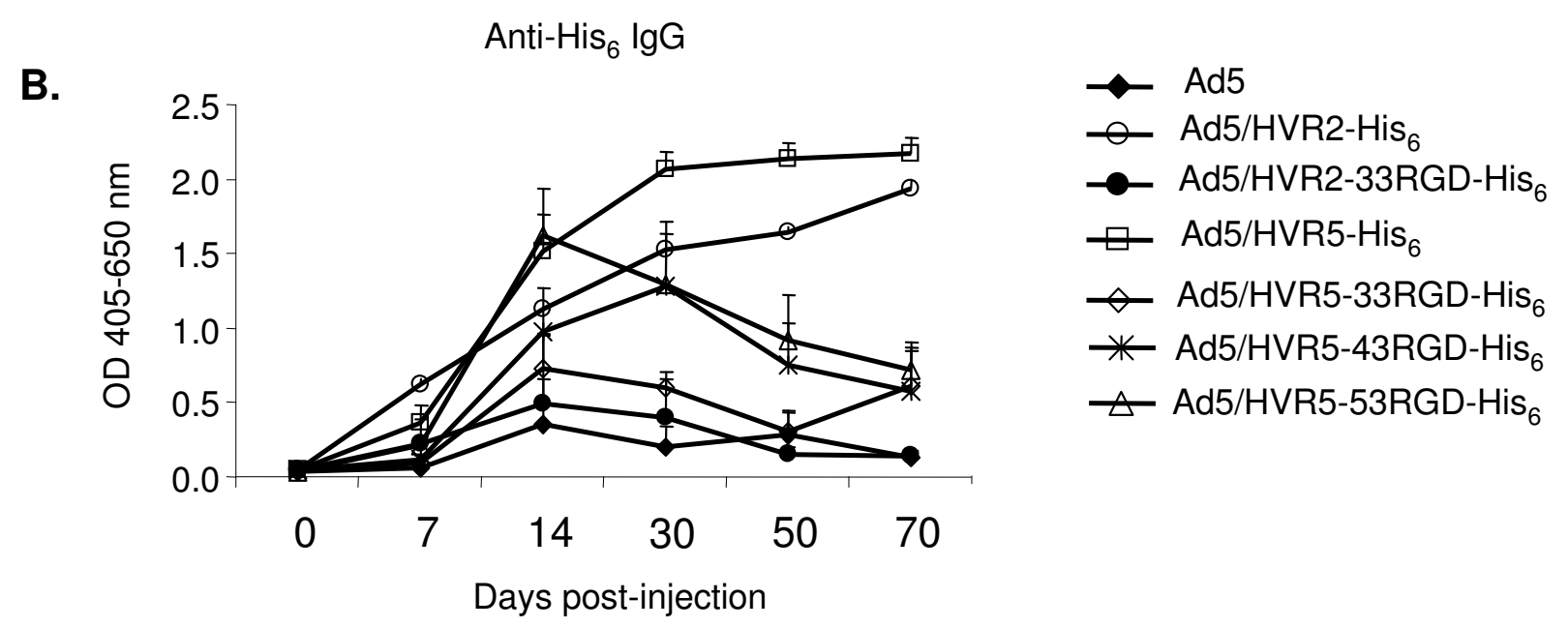

Figure 3

Capsid-incorporated antigens elicit an IgG immune response. C57BL/6] mice were immunized with $10^{10} \mathrm{VP}$ of $\mathrm{Ad}$ vectors. Post-immunization sera were collected after (A) 30 days post-injection or (B) 0-70 days post-injection for ELISA binding assays. $20 \mu \mathrm{M}$ of synthesized antigenic peptide His ${ }_{6}$ peptide was bound to ELISA plates. Residual unbound peptide was washed from the plates. The plates were then incubated with immunized mice sera, the binding was detected with IgG-specific HRPconjugated anti-mouse secondary antibody. OD absorbance represents the sera levels of antibodies. Values are expressed as the mean \pm standard error of three replicates. $*$ indicates a $\mathrm{P}$ value of $<.05$., $* * \mathrm{P}<.00 \mathrm{I}, * * * \mathrm{P}<.0000 \mathrm{I}$. Control viruses are Ad5, Ad/HVR2-His 6 and Ad/HVR5-His . 


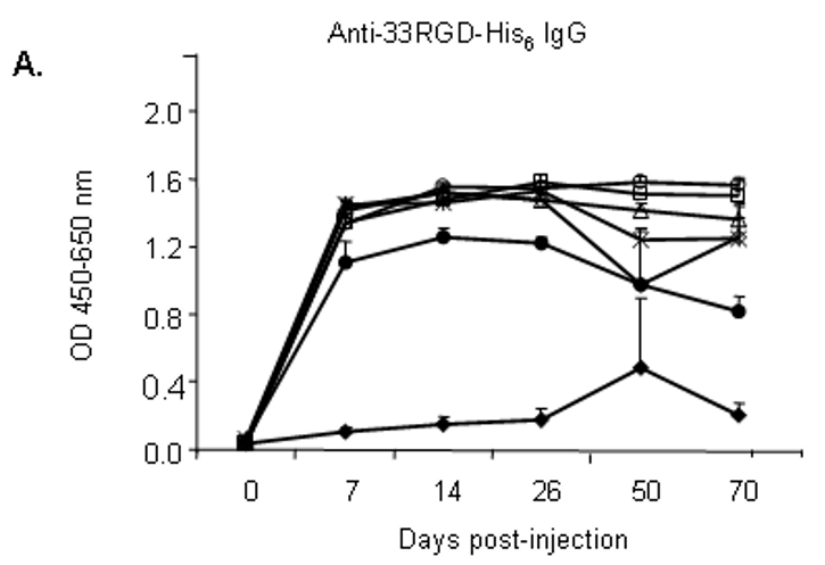

B.

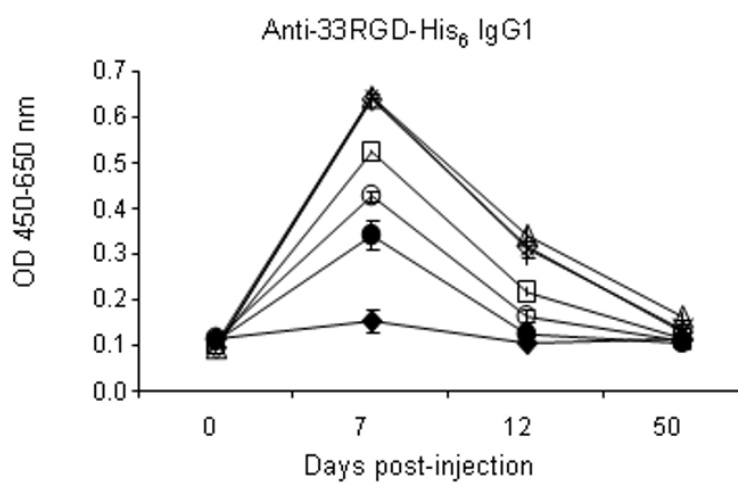

C.
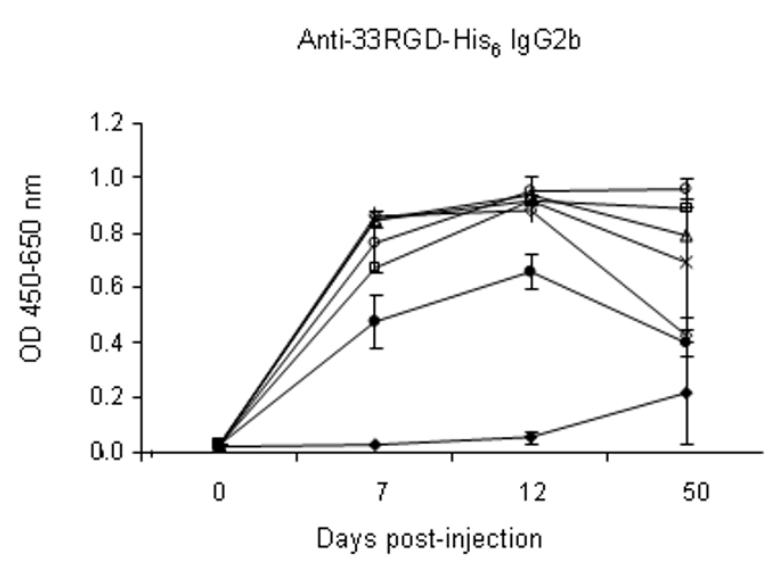

D.

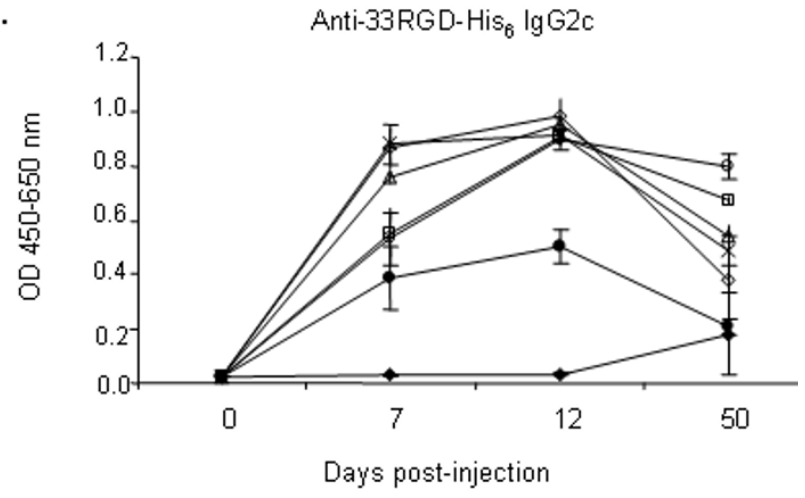

Figure 4

Capsid-incorporated antigens elicit a varied immune response. (A-D) C57BL/6] mice were immunized with $10^{10} \mathrm{VP}$ of Ad vectors. Post-immunization sera were collected after 50 days post-injection for ELISA binding assays. $20 \mu M$ of synthetic peptide $33 R G D-H_{i}{ }_{6}\left(R G D\right.$ residue flanked by $\mathrm{His}_{6} /$ Linker) was bound to the plate. The plates were then incubated with immunized mice sera, the binding was detected with isotype-specific HRP-conjugated anti-mouse secondary antibody (A, lgG; B, $\lg \mathrm{I}$; $C, \lg G 2 \mathrm{~b} ; \mathrm{D}, \lg G 2 \mathrm{c})$. OD absorbance represents the sera levels of antibodies. Values are expressed as the mean \pm standard error of three replicates. Control viruses are Ad5, Ad/HVR2-His 6 and Ad/HVR5-His 6 . 
A.

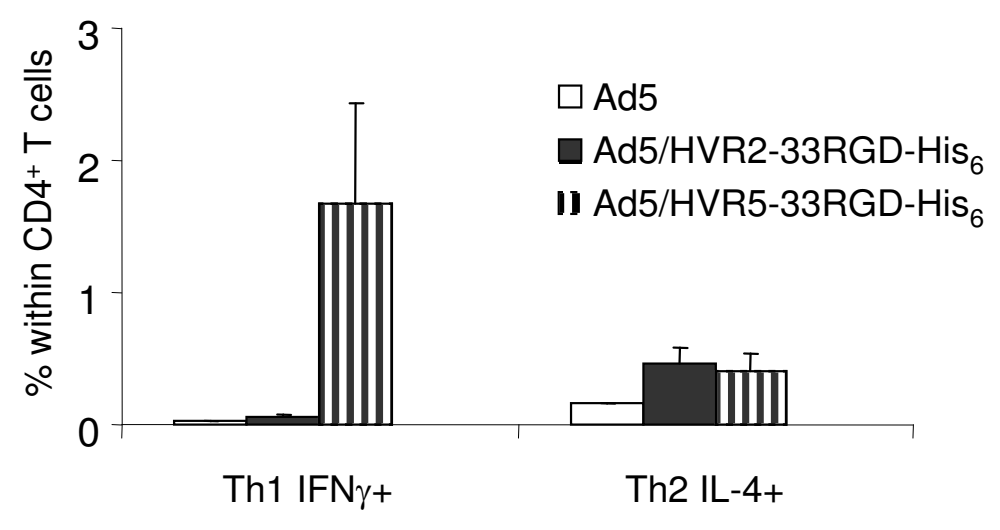

B.

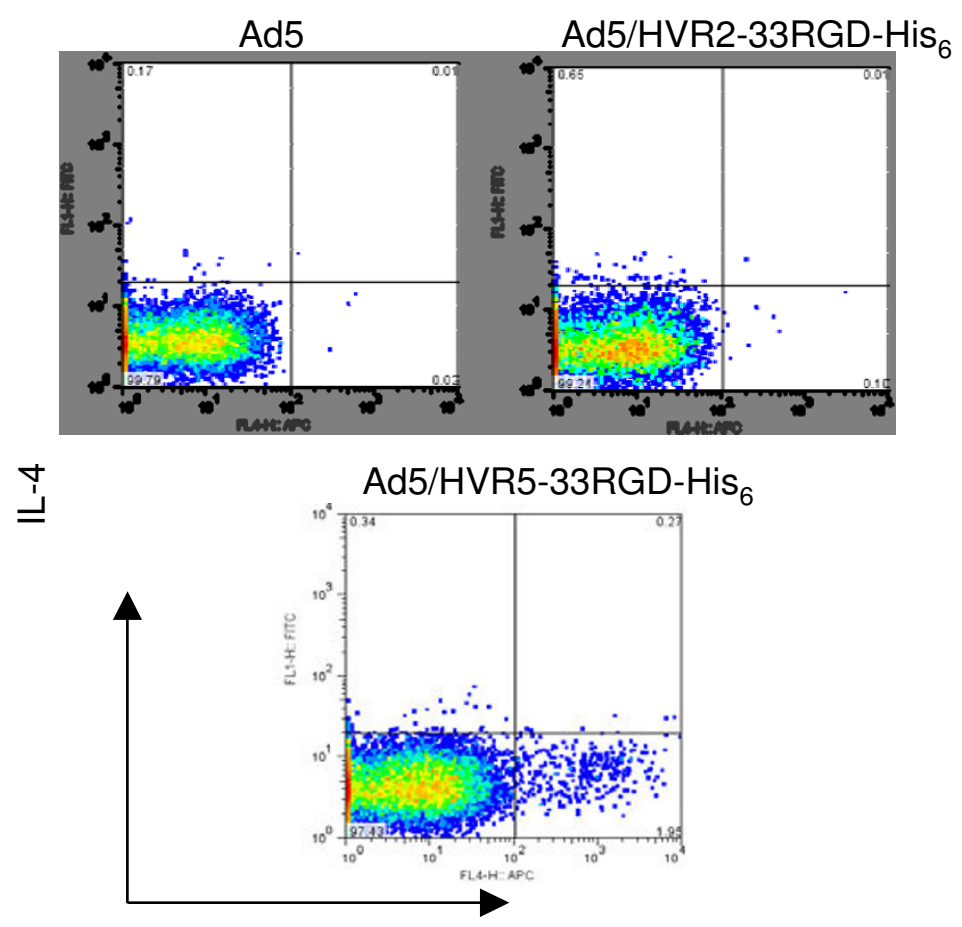

IFN- $\gamma$

\section{Figure 5}

Capsid-incorporated antigens elicit a varied T cell response. (A-B) C57BL/6) mice were immunized with $10^{10} \mathrm{VP}$ of Ad vectors. On day 40 , these mice were intravenously boosted with the same dose of the same vectors. A single-cell suspension of spleen cells was prepared on day 9 after secondary virus infection. Cells were stained with a fluorescent labeled anti-CD4 antibody and then permeabilized in intracellular stain with fluorescent conjugated antibodies against IL- 4 or IFN- $\gamma$. Samples were acquired on a FACSCalibur and data were analyzed with Flowjo software. Values are expressed as the mean \pm standard error of three replicates. 
Ad5/HVR5-33RGD-His ${ }_{6}$ (Fig. 5A-B). The increased IgG antibody response to 33RGD-His ${ }_{6}$ in the HVR5 loop of Ad is associated with a significant increased Th1 T cell response.

To evaluate whether immunization with our hexon-modified viruses resulted in improved secondary antibody responses, mice were immunized with Ad5, Ad5/HVR2$33 \mathrm{RGD}^{-\mathrm{His}_{6}}$, or Ad5/HVR5-33RGD-His $_{6}$. Forty days later, mice were boosted with the respective hexon-modified viruses. Sera levels of antibodies against the 33RGD-His ${ }_{6}$ peptide were determined at day 9 following the booster injection. The Ad5/HVR5-33RGD group exhibited further enhancement with respect to IgG1 antibody response to the 33RGD-His ${ }_{6}$ peptide after boosting compared to Ad5
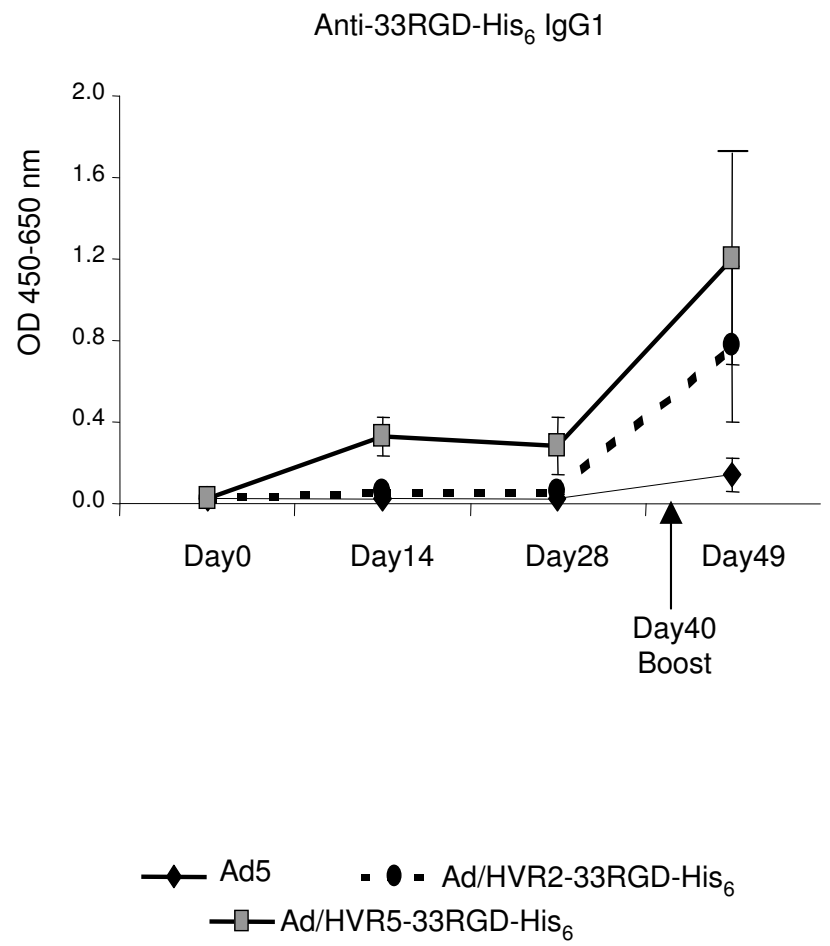

\section{Figure 6}

Repeat administration of hexon-modified viruses results in boosting of the anti-33RGD-His ${ }_{6}$ immune response. $C 57 \mathrm{BL} / 6 \mathrm{~J}$ mice were immunized with 1010VP of Ad vectors. On day 40 , these mice were intravenously boosted with the same dose of the same vectors. Post-immunization sera were collected after 9 days post-injection for ELISA binding assays. $20 \mu \mathrm{M}$ of synthetic peptide 33RGD$\mathrm{His}_{6}$ was bound to the plate. The plates were then incubated with immunized mice sera, the binding was detected with isotype-specific HRP-conjugated anti-mouse secondary antibody. OD absorbance represents the sera levels of antibodies. Values are expressed as the mean \pm standard error of three replicates. control (Fig. 6). This trend is similar to that seen after primary immunization (Fig. 4B).

\section{Discussion}

We have developed novel adenovirus vectors that have the potential to optimize adenovirus vaccine approaches. This strategy involves inserting antigenic epitopes of various sizes into HVR2 or HVR5 regions of the Ad capsid protein, hexon, to stimulate epitope-specific antibody responses following vaccination. The ability to insert multiple antigens in the Ad capsid will allow vaccination with antigenic epitopes in one vector. This method offers the ability to compare a range of identical epitopes incorporated within HVRs for antigenic optimization. Our current study is the first study of its kind to compare a range of identical epitopes incorporated within HVRs for antigenic optimization. Importantly, our data ascribe a maximal antigenic incorporation size at HVR2 and HVR5 as it relates to identical antigenic epitopes.

Similar studies have been performed by other groups, Worgall and colleagues describe incorporations of a neutralizing epitope from the Pseudomonas aeruginosa outer membrane protein $\mathrm{F}$ (OprF) into adenovirus HVR5 [10]. The authors showed an increase in antibody response in BALB/c mice consisting of both IgG1 and IgG2a subtypes. Additionally, when mice immunized with the virus containing the OprF epitope were subjected to pulmonary challenge with $P$. aeruginosa, 60 to $80 \%$ survival was achieved. This was in contrast to results seen by McConnell et.al, who published that chimeric hexons containing incorporations of B. anthracis protective antigen (PA) elicited antibodies against PA in mice but failed to yield protection against anthrax toxin (lethal factor) challenge [15]. The authors speculate that the varying results reflect a difference in the ability of the selected epitopes to elicit a neutralizing response in the varying disease models or a difference in the antibody titers necessary to achieve protection against $P$. aeruginosa compared to lethal factor challenge. In addition, they speculate that the latter may be related to the fact that in the anthrax model the response is directed against a secreted bacterial toxin, while in the $P$. aeruginosa model the response is directed against the bacterium itself. Similar studies have been performed by Krause et. al, [14]. Krause's study compared the immune response generated by incorporating the hemagglutinin (HA) protein of the influenza A virus incorporated into the outer Ad capsid protein hexon, penton base, fiber knob, or protein IX. The HA epitope was recognized by the anti-HA antibody in all four modified virions with slightly stronger binding to the HA presented in hexon HVR5. However, this study does not investigate whether the size of the incorporated epitopes could also affect the immune response generated. 
The strategy we pursued involved the genetic incorporation into hexon HVR2 and 5, respectively. We chose the RGD-containing motif to incorporate into the hexon protein because the RGD motif has been demonstrated to have a critical role in Ad entry. Thus by incorporating this molecule into the Ad hexon we speculated that it might be possible to enhance Ad viral tropism. [17]. In addition, we have previously established that these RGD motifs can be inserted into another Ad capsid protein fiber, thus modulating vector tropism [22]. A total of six fragments of the penton base protein ranging in size from 33 to 83 aa were incorporated into the Ad5 hexon HVR2 or HVR5. Viable viruses were produced with incorporations of 33 aa at HVR2 and up to 53 aa at HVR5 (Table 2A). To effectively invoke an epitope-specific immune response, genetically incorporated epitopes must be accessible on the Ad surface. This study illustrates that RGD-His ${ }_{6}$ motifs incorpo- $^{-}$ rated within HVR2 or HVR5 were accessible on the adenovirus surface based on anti-His ${ }_{6}$ ELISA (Fig. 2). There was no significant difference between in vitro antibody binding of viruses that contain $\mathrm{His}_{6}$ residues at HVR2 or HVR5, or viruses that contain the 33 RGD-His ${ }_{6}$ epitope at HVR2 or HVR5. This finding confirms that the 33RGD-His ${ }_{6}$ motifs incorporated within HVR2 or HVR5 are indeed accessible on the Ad surface and should therefore be available to antibodies in vivo. We observed that increasing the size of incorporations at hexon HVRs increased the virological viral particle/infectious particle ratios (Table 3), we speculate that virus assembly and stability is affected. In addition, we have observed more aggregation with inserts incorporated at the Ad hexon HVR5 locale, we also further speculate that insertions containing RGD epitopes lend to virus aggregation. Since modifications to Ad capsid proteins can influence infectivity as well as immunogenicity of Ad vaccines and transduction efficiency, it is possible that our modifications would significantly alter the infectivity of Ad. Ad infectivity occurs through the binding of the Ad capsid proteins penton base and fiber to cellular receptors [30-33]. More recently, hexon HVR's have been implicated in liver transduction [19-21]. We speculate that these recent findings by kalyuzhniy and colleagues, indicate that our Ad vectors are more clinically relevant due to the likelihood of less liver transduction.

Successful stimulation of immune responses by Ad vaccines schemas are thought to be dependent partly on the activation of antigen presenting cells, particularly dendritic cells [34,35]. Indeed, genetic modifications made to the capsid in this present study impair some virological properties such as virus particle/infectious particle ratios and gene transfer efficacies (data not shown), but our data indicates that in vivo immune response was not affected. However, we will pursue investigation regarding the uptake of our hexon-modified virus by antigen presenting cells. Of note, in this study we notice higher in vivo immune response of viruses containing 43 or 53 RGD$\mathrm{His}_{6}$ epitopes at HVR5 compared to that of 33RGD-His ${ }_{6}$. Sequence analysis of these three epitopes show no obvious reason for this trend (ie. hydrophobic or hydrophilic patterns), therefore; detailed structural analysis must be performed.

Finally, our results indicate that mice boosted with Ad5/ HVR2-33RGD-His ${ }_{6}$ or Ad5/HVR5-33RGD-His ${ }_{6}$ produced an improved secondary immune response as compared to the control Ad5 vector (Fig. 6). Successful boosting is an important factor because anti-Ad exposure after administration of Ad vectors does not generally allow repeat administration with an Ad vector of the same serotype $[36-40,10]$. Anti-Ad immunity is thought to be an obstacle for the use of Ad as a gene therapy vector; re-administration of the same vector would be beneficial in the development of Ad-based vaccines to enable boosting of antigen-specific immune response. In our study, repeat immunization resulted in boosting of the anti-33RGD$\mathrm{His}_{6}$ antibody responses. The Ad5/HVR5-33RGD-His vector exhibited the highest antibody response to both 33RGD-His ${ }_{6}$ peptide and $\mathrm{His}_{6}$ peptide (data not shown) after boosting; therefore the Ad5/HVR5-33RGD-His ${ }_{6}$ vector is the best construct to generate the Ad vaccine response with respect to our model antigens.

Our study in contrast, to other reports illustrates the qualitative differences with respect to incorporation of large epitopes within HVR2 or HVR5, until now most reports only investigate HVR5 as a potential incorporation locale. Our study demonstrates that HVR5 is more permissible than HVR2 with respect to incorporation of our largest model antigen. Immunizations with vectors that present smaller $\mathrm{His}_{6}$ insertions at HVR2 compared to HVR5, yield similar results with respect to antibody response and insertion locale. In contrast, immunizations with viruses containing large insertions at HVR5 yielded higher antibody and Th1 responses compared to insertions at HVR2. These results were in contrast to that seen with in vitro ELISA assays, which were equal binding of insertions at HVR2 or 5 independent of insertion size (Fig. 2). Furthermore, it is likely that large insertions at HVR2 are not permissible due to the surrounding Ad protein structure/ environment. However, smaller inserts may be tolerated at HVR2.

We plan to investigate factors limiting insertions at HVR2 and HVR5 by means of cryoEM analysis, this work will correlate well with the Ad crystal structure and cryoEM analysis which has been recently solved [41-44]. In the aggregate, our study demonstrates that utilization of the HVR2 or 5 locales predicate optimal antigen size and configuration. Based on this technology, we will be able to 
establish the critical correlates between antigen locale/ accessibility within the capsid context and vaccine efficacy. Our study evaluated model antigens at HVR2 or HVR5; further studies are necessary to evaluate therapeutic antigens at these locales in the context of binding and antibody neutralization. Transitioning our dual hexon presentation platform to present therapeutic antigens will also allow us to evaluate and use challenge models for efficacy and antigen protection assays. Capsid incorporation of antigens is a highly innovative strategy to present antigens in the context of adenovirus vaccine schemas. This strategy can also be exploited to construct multivalent vaccines, which can allow vaccination against multiple strains of a particular infectious disease or protection against multiple unrelated diseases. Of particular interest to us is the potential to expand our dual hexon antigen presentation strategy to develop Ad-based vaccinations against HIV infection and many other infections or diseases.

\section{Abbreviations}

Ad: Adenovirus; Ad5: Adenovirus serotype 5; aa: Amino acid; ELISA: Enzyme-linked immunosorbent assay; HA: Hemagglutinin; His: Histidine; His $_{6}$ : Six-histidine; HVRs: Hypervariable regions; IP: Infectious particles; RGD: ArgGly-Asp; VP: Viral particles.

\section{Acknowledgements}

The authors would also like to acknowledge Dr. Maaike Everts and Erin E. Thacker as well as Yizhe Tang for their critical reading of the manuscript. This work was supported by grants from the National Institutes of Health: 5T32AI07493-II (Dr. Casey Morrow), and IR2IAI076096-0I (Dr. David T. Curiel).

\section{References}

I. Jooss K, Chirmule N: Immunity to adenovirus and adeno-associated viral vectors: implications for gene therapy. Gene The 2003, 10:955-963

2. Dmitriev I, Krasnykh V, Miller CR, Wang M, Kashentseva E, Mikheeva $\mathrm{G}$, Belousova N, Curiel DT: An adenovirus vector with genetically modified fibers demonstrates expanded tropism via utilization of a coxsackievirus and adenovirus receptorindependent cell entry mechanism. J Virol 1998, 72:9706-9713.

3. Barnett BG, Crews CJ, Douglas JT: Targeted adenoviral vectors. Biochim Biophys Acta 2002, I 575: I- 14

4. Kay MA, Glorioso JC, Naldini L: Viral vectors for gene therapy: the art of turning infectious agents into vehicles of therapeutics. Nat Med 200I, 7:33-40.

5. Randrianarison-Jewtoukoff $V$, Perricaudet M: Recombinant adenoviruses as vaccines. Biologicals $1995,23: 145-157$

6. Wilson JM: Adenoviruses as gene-delivery vehicles. $N$ Engl Med 1996, 334: I 185-I I87.

7. Sullivan NJ, Sanchez A, Rollin PE, Yang ZY, Nabel G): Development of a preventive vaccine for Ebola virus infection in primates. Nature 2000, 408:605-609.

8. Nabel G]: Challenges and opportunities for development of an AIDS vaccine. Nature 2001, 41 0:1002-1007.

9. Gomez-Roman VR, Robert-Guroff M: Adenoviruses as vectors for HIV vaccines. AIDS Rev 2003, 5:178-185.

10. Worgall S, Krause A, Rivara M, Hee KK, Vintayen EV, Hackett NR, Roelvink PW, Bruder JT, Wickham TJ, Kovesdi I, Crystal RG: Protection against $P$. aeruginosa with an adenovirus vector containing an OprF epitope in the capsid. J Clin Invest 2005, I 15:1281-1289.
II. Boyer JL, Kobinger G, Wilson JM, Crystal RG: Adenovirus-based genetic vaccines for biodefense. Hum Gene Ther 2005, 16:157-168.

12. Barouch DH, Nabel G]: Adenovirus vector-based vaccines for human immunodeficiency virus type I. Hum Gene Ther 2005 , 16:149-156.

13. Crompton J, Toogood Cl, Wallis N, Hay RT: Expression of a foreign epitope on the surface of the adenovirus hexon. J Gen Virol I994, 75 ( Pt I): I33- I39.

14. Krause A, Joh JH, Hackett NR, Roelvink PW, Bruder JT, Wickham TJ, Kovesdi I, Crystal RG, Worgall S: Epitopes expressed in different adenovirus capsid proteins induce different levels of epitopespecific immunity. J Virol 2006, 80:5523-5530.

15. McConnell MJ, Danthinne X, Imperiale MJ: Characterization of a permissive epitope insertion site in adenovirus hexon. J Virol 2006, 80:536I-5370.

16. Worgall S, Krause A, Qiu J, Joh J, Hackett NR, Crystal RG: Protective immunity to pseudomonas aeruginosa induced with a capsid-modified adenovirus expressing P. aeruginosa OprF. J Virol 2007, 8 I: I380|- I 3808.

17. Vigne E, Mahfouz I, Dedieu JF, Brie A, Perricaudet M, Yeh P: RGD inclusion in the hexon monomer provides adenovirus type 5 based vectors with a fiber knob-independent pathway for infection. J Virol 1999, 73:5156-5161.

18. Wu H, Han T, Belousova N, Krasnykh V, Kashentseva E, Dmitriev I, Kataram M, Mahasreshti PJ, Curiel DT: Identification of sites in adenovirus hexon for foreign peptide incorporation. J Virol 2005, 79:3382-3390.

19. Kalyuzhniy O, Di Paolo NC, Silvestry M, Hofherr SE, Barry MA, Stewart PL, Shayakhmetov DM: Adenovirus serotype $\mathbf{5}$ hexon is critical for virus infection of hepatocytes in vivo. Proc Natl Acad Sci U S A 2008, 105:5483-5488.

20. Waddington SN, McVey JH, Bhella D, Parker AL, Barker K, Atoda H, Pink R, Buckley SM, Greig JA, Denby L, Custers J, Morita T, Francischetti IM, Monteiro RQ, Barouch DH, van RN, Napoli C, Havenga MJ, Nicklin SA, Baker AH: Adenovirus serotype $\mathbf{5}$ hexon mediates liver gene transfer. Cell 2008, 132:397-409.

21. Vigant F, Descamps D, Jullienne B, Esselin S, Connault E, Opolon P, Tordjmann T, Vigne E, Perricaudet M, Benihoud K: Substitution of hexon hypervariable region 5 of adenovirus serotype 5 abrogates blood factor binding and limits gene transfer to liver. Mol Ther 2008, I 6: |474- |480.

22. Belousova N, Krendelchtchikova V, Curiel DT, Krasnykh V: Modulation of adenovirus vector tropism via incorporation of polypeptide ligands into the fiber protein. J Virol 2002 76:862I-863।.

23. Wu H, Dmitriev I, Kashentseva E, Seki T, Wang M, Curiel DT: Construction and characterization of adenovirus serotype 5 packaged by serotype 3 hexon. J Virol 2002, 76: I 2775-12782.

24. Wu H, Seki T, Dmitriev I, Uil T, Kashentseva E, Han T, Curiel DT: Double modification of adenovirus fiber with RGD and polylysine motifs improves coxsackievirus-adenovirus receptorindependent gene transfer efficiency. Hum Gene Ther 2002, 13:1647-1653.

25. Zhang HG, High KA, Wu Q, Yang P, Schlachterman A, Yu S, Yi N, Hsu HC, Mountz JD: Genetic analysis of the antibody response to AAV2 and factor IX. Mol Ther 2005, I I:866-874

26. Harrington LE, Hatton RD, Mangan PR, Turner H, Murphy TL, Murphy KM, Weaver CT: Interleukin I7-producing CD4+ effector $T$ cells develop via a lineage distinct from the $T$ helper type I and 2 lineages. Nat Immunol 2005, 6: I I 23- I I 32

27. Mangan PR, Harrington LE, O'Quinn DB, Helms WS, Bullard DC, Elson CO, Hatton RD, Wahl SM, Schoeb TR, Weaver CT: Transforming growth factor-beta induces development of the T(H) I 7 lineage. Nature 2006, 44 I:23 I-234.

28. Asnagli H, Murphy KM: Stability and commitment in $\mathbf{T}$ helper cell development. Curr Opin Immunol 200 I, 13:242-247.

29. Stevens TL, Bossie A, Sanders VM, Fernandez-Botran R, Coffman RL, Mosmann TR, Vitetta ES: Regulation of antibody isotype secretion by subsets of antigen-specific helper T cells. Nature 1988 , 334:255-258

30. Hong SS, Karayan L, Tournier J, Curiel DT, Boulanger PA: Adenovirus type 5 fiber knob binds to MHC class I alpha2 domain at the surface of human epithelial and B lymphoblastoid cells. EMBO J 1997, 16:2294-2306. 
31. Mercier S, Rouard H, fau-Larue MH, Eloit M: Specific antibodies modulate the interactions of adenovirus type 5 with dendritic cells. Virology 2004, 322:308-317.

32. Wickham TJ, Mathias P, Cheresh DA, Nemerow GR: Integrins alpha $v$ beta 3 and alpha $v$ beta 5 promote adenovirus internalization but not virus attachment. Cell 1993, 73:309-319.

33. Worgall S, Busch A, Rivara M, Bonnyay D, Leopold PL, Merritt R, Hackett NR, Rovelink PW, Bruder JT, Wickham TJ, Kovesdi I, Crystal RG: Modification to the capsid of the adenovirus vector that enhances dendritic cell infection and transgene-specific cellular immune responses. J Virol 2004, 78:2572-2580.

34. Labow D, Lee S, Ginsberg RJ, Crystal RG, Korst RJ: Adenovirus vector-mediated gene transfer to regional lymph nodes. Hum Gene Ther 2000, I I:759-769.

35. Zhang Y, Chirmule N, Gao GP, Qian R, Croyle M, Joshi B, Tazelaar J, Wilson JM: Acute cytokine response to systemic adenoviral vectors in mice is mediated by dendritic cells and macrophages. Mol Ther 200I, 3:697-707.

36. Jooss K, Yang Y, Fisher KJ, Wilson JM: Transduction of dendritic cells by DNA viral vectors directs the immune response to transgene products in muscle fibers. / Virol I 998, 72:42 I 2-4223.

37. Hackett NR, Kaminsky SM, Sondhi D, Crystal RG: Antivector and antitransgene host responses in gene therapy. Curr Opin Mol Ther 2000, 2:376-382.

38. Mack CA, Song WR, Carpenter H, Wickham TJ, Kovesdi I, Harvey BG, Magovern CJ, Isom OW, Rosengart T, Falck-Pedersen E, Hackett NR, Crystal RG, Mastrangeli A: Circumvention of anti-adenovirus neutralizing immunity by administration of an adenovira vector of an alternate serotype. Hum Gene Ther 1997, 8:99-109.

39. Mastrangeli A, Harvey BG, Yao J, Wolff G, Kovesdi I, Crystal RG, Falck-Pedersen E: "Sero-switch" adenovirus-mediated in vivo gene transfer: circumvention of anti-adenovirus humoral immune defenses against repeat adenovirus vector administration by changing the adenovirus serotype. Hum Gene Ther 1996, 7:79-87.

40. Yang Y, Li Q, Ertl HC, Wilson JM: Cellular and humoral immune responses to viral antigens create barriers to lung-directed gene therapy with recombinant adenoviruses. J Virol 1995, 69:2004-2015.

4I. Roberts MM, White JL, Grutter MG, Burnett RM: Three-dimensional structure of the adenovirus major coat protein hexon. Science 1986, 232:I|48-II5I.

42. Saban SD, Nepomuceno RR, Gritton LD, Nemerow GR, Stewart PL: CryoEM structure at 9A resolution of an adenovirus vector targeted to hematopoietic cells. I Mol Biol 2005, 349:526-537.

43. Xia D, Henry LJ, Gerard RD, Deisenhofer J: Crystal structure of the receptor-binding domain of adenovirus type 5 fiber protein at I.7 A resolution. Structure 1994, 2: 1259-1270.

44. Zubieta C, Schoehn G, Chroboczek J, Cusack S: The structure of the human adenovirus 2 penton. Mol Cell 2005, I7: I2I-I35.
Publish with Bio Med Central and every scientist can read your work free of charge

"BioMed Central will be the most significant development for disseminating the results of biomedical research in our lifetime. "

Sir Paul Nurse, Cancer Research UK

Your research papers will be:

- available free of charge to the entire biomedical community

- peer reviewed and published immediately upon acceptance

- cited in PubMed and archived on PubMed Central

- yours - you keep the copyright

Submit your manuscript here:

http://www.biomedcentral.com/info/publishing_adv.asp
BioMedcentral 